\title{
Antioxidant potential and Angiotensin-Converting Enzyme (ACE) inhibitory activity of orotic acid-loaded gum arabic nanoparticles
}

\begin{abstract}
Orotic acid (OA) nanoparticles were prepared using the freeze-drying method. The antihypertensive activity and antioxidant capacity of OA and orotic acid-loaded gum arabic nanoparticles (OAGANPs) were examined using the angiotensin-converting enzyme (ACE), 1,1-diphenyl-2-picrylhydrazyl (DPPH), nitric oxide (NO), and $\beta$-carotene assays, as well as the quantification of total phenolic content (TPC). The DPPH and NO scavenging activities of OAGANPs were significantly higher than those of the OA solution. The $\beta$-carotene bleaching assay of OAGANPs showed a dose-dependent trend, while $500 \mu \mathrm{g} / \mathrm{ml}$ was significantly more effective than the other concentrations, which exerted $63.4 \%$ of the antioxidant activity. The in vitro antihypertensive assay revealed that the OAGANPs exhibited the most potent ACE inhibition activity, when compared to the OA solution. Hence, results revealed the potential of preparing the $\mathrm{OA}$ as a nanoparticle formulation in enhancing the antioxidant and antihypertensive properties compared to the OA solution.
\end{abstract}

Keyword: Antihypertensive; Antioxidant; Nanoparticles; Radical scavenging 